\title{
Cycle-CNN for Colorization towards Real Monochrome-Color Camera Systems
}

\author{
Xuan Dong, ${ }^{1}$ Weixin $\mathrm{Li}^{2 *}$ Xiaojie Wang, ${ }^{1}$ Yunhong Wang ${ }^{2}$ \\ ${ }^{1}$ School of Computer Science, Beijing University of Posts and Telecommunications, Beijing, 100876 \\ ${ }^{2}$ Beijing Advanced Innovation Center for Big Data and Brain Computing, Beihang University, Beijing, 100191 \\ \{dongxuan8811, xjwang\}@ bupt.edu.cn, \{weixinli, yhwang\}@ buaa.edu.cn
}

\begin{abstract}
Colorization in monochrome-color camera systems aims to colorize the gray image $\mathbf{I}_{\mathbf{G}}$ from the monochrome camera using the color image $\mathbf{R}_{\mathbf{C}}$ from the color camera as reference. Since monochrome cameras have better imaging quality than color cameras, the colorization can help obtain higher quality color images. Related learning based methods usually simulate the monochrome-color camera systems to generate the synthesized data for training, due to the lack of ground-truth color information of the gray image in the real data. However, the methods that are trained relying on the synthesized data may get poor results when colorizing real data, because the synthesized data may deviate from the real data. We present a new CNN model, named cycle CNN, which can directly use the real data from monochrome-color camera systems for training. In detail, we use the colorization $\mathrm{CNN}$ model to do the colorization twice. First, we colorize $\mathbf{I}_{\mathbf{G}}$ using $\mathbf{R}_{\mathbf{C}}$ as reference to obtain the first-time colorization result $\mathbf{I}_{\mathbf{C}}$. Second, we colorize the de-colored map of $\mathbf{R}_{\mathbf{C}}$, i.e. $\mathbf{R}_{\mathbf{G}}$, using the first-time colorization result $\mathbf{I}_{\mathbf{C}}$ as reference to obtain the second-time colorization result $\mathbf{R}_{\mathbf{C}}^{\prime}$. In this way, for the second-time colorization result $\mathbf{R}_{\mathbf{C}}^{\prime}$, we use the original color map $\mathbf{R}_{\mathbf{C}}$ as ground-truth and introduce the cycle consistency loss to push $\mathbf{R}_{\mathbf{C}}^{\prime} \approx \mathbf{R}_{\mathbf{C}}$. Also, for the first-time colorization result $\mathbf{I}_{\mathbf{C}}$, we propose a structure similarity loss to encourage the luminance maps between $\mathbf{I}_{\mathbf{G}}$ and $\mathbf{I}_{\mathbf{C}}$ to have similar structures. In addition, we introduce a spatial smoothness loss within the colorization $\mathrm{CNN}$ model to encourage spatial smoothness of the colorization result. Combining all these losses, we could train the colorization $\mathrm{CNN}$ model using the real data in the absence of the ground-truth color information of $\mathbf{I}_{\mathbf{G}}$. Experimental results show that we can outperform related methods largely for colorizing real data.
\end{abstract}

\section{Introduction}

With the increasing use of monochrome-color camera systems in high-end smart phones, e.g. Huawei P30, Mate30, etc., the colorization problem within these systems is attracting more and more attentions from the academic and industrial communities.

As shown in Fig. 1, colorization in monochrome-color camera systems aims to colorize the gray image $\mathbf{I}_{\mathbf{G}}$ from

\footnotetext{
${ }^{*}$ Corresponding Author. Copyright (C) 2020, Association for the Advancement of Artificial Intelligence (www.aaai.org). All rights reserved.
}
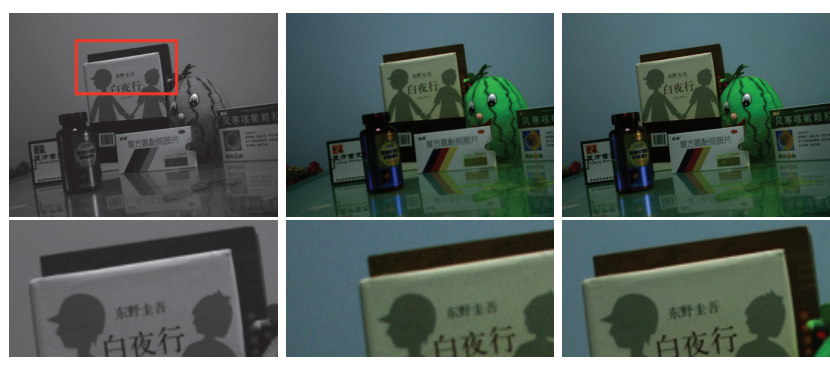

(a) Input pair of gray image $\mathbf{I}_{\mathbf{G}}$ and color image $\mathbf{R}_{\mathbf{C}}$. (b) Our colorization result $\mathbf{I}_{\mathbf{C}}^{*}$.

Figure 1: The input pair of gray image $\mathbf{I}_{\mathbf{G}}$ and color image $\mathbf{R}_{\mathbf{C}}$ are shot by the monochrome and color cameras, respectively. By directly using these real data for training, our algorithm learns to colorize $\mathbf{I}_{\mathbf{G}}$ using $\mathbf{R}_{\mathbf{C}}$ as reference.

the monochrome camera using the color image $\mathbf{R}_{\mathbf{C}}$ from the color camera as reference. Between the monochrome and color cameras, there exist different hardwares, e.g. the color filter array, and different software modules, e.g. white balance, demosaic, etc. As a result, on the one hand, the monochrome camera has better light efficiency (Jeon et al. 2016; Dong et al. 2019) than the color camera, and thus the gray image has higher quality, i.e. signal-noise ratio, than the color image. This motivates researchers to do the colorization so as to get higher quality color images using the monochrome-color camera systems. On the other hand, the pair of gray and color images have different luminance, blur, noises, etc. These cause the difficulty for the colorization.

Among existing methods for colorization within the monochrome-color camera system, some are traditional hand-crafted methods, e.g. (Jeon et al. 2016). With the successful use of deep learning in various computer vision problems, some deep learning based methods, e.g. (Dong et al. 2019) are proposed recently, which have shown to be able to obtain higher accuracy than the traditional ones. However, in the deep learning methods, e.g. (Dong et al. 2019), the models usually need ground-truth color information of the input gray images as annotations for training. Due to the lack of ground-truth color information in the real data, as shown in Fig. 2, current methods, e.g. (Jeon et al. 2016; Dong et al. 2019), usually synthesize data to simulate the 


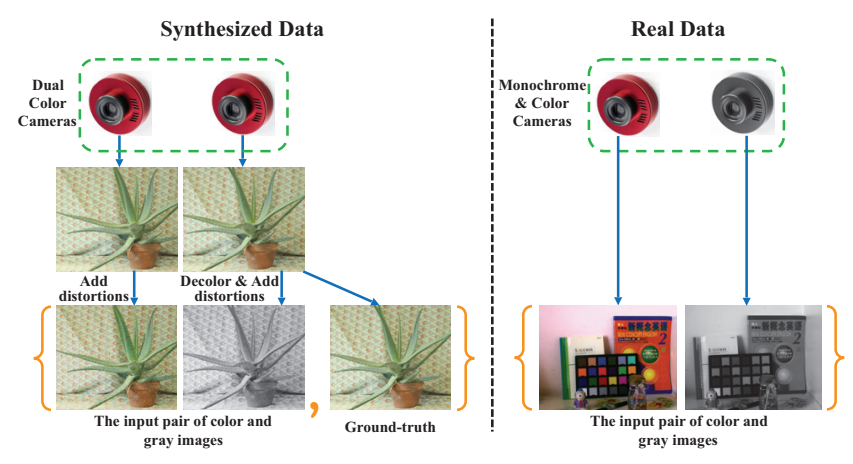

Figure 2: How the synthesized data and the real data are obtained. The real data are the pair of gray and color images shot from the monochrome-color camera system. The synthesized data are the pair of gray and color images that are synthesized using a pair of color images from the dual-color camera system.

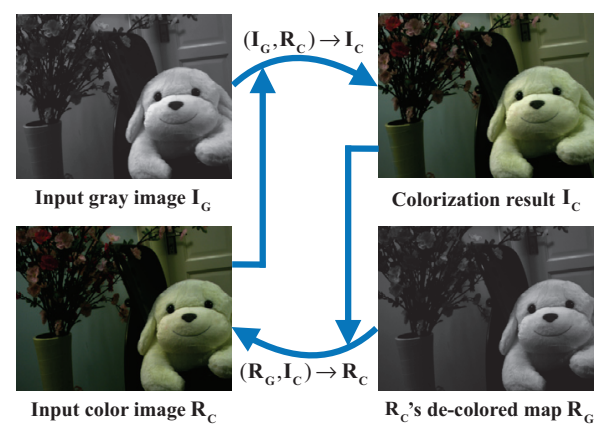

Figure 3: The insight of cycle colorization consistency. When doing the colorization twice, i.e. firstly $\left(\mathbf{I}_{\mathbf{G}}, \mathbf{R}_{\mathbf{C}}\right) \rightarrow$ $\mathbf{I}_{\mathbf{C}}$ and secondly $\left(\mathbf{R}_{\mathbf{G}}, \mathbf{I}_{\mathbf{C}}\right) \rightarrow \mathbf{R}_{\mathbf{C}}^{\prime}$, the second-time colorization result $\mathbf{R}_{\mathbf{C}}^{\prime}$ should arrive back at $\mathbf{R}_{\mathbf{C}}$.

real data from the monochrome-color camera system. However, the degradation models for synthesizing the data may deviate from the ones in real imaging systems within the monochrome and color cameras. Thus, the synthesized data could hardly simulate the real data perfectly. As a result, the deep learning methods, which are trained relying on the synthesized data, may have very poor results when colorizing the real data.

To overcome this limitation, in this paper, we propose a new convolutional neural network (CNN) model and aim to directly use the real data from the monochrome-color camera system for training.

Our insight is based on the property of cycle colorization consistency. As shown in Fig. 3, when we do the colorization twice, i.e. firstly colorizing $\mathbf{I}_{\mathbf{G}}$ using $\mathbf{R}_{\mathbf{C}}$ as reference and secondly colorizing the gray map of $\mathbf{R}_{\mathbf{C}}$, i.e. $\mathbf{R}_{\mathbf{G}}$, using the obtained first-time colorization result $\mathbf{I}_{\mathbf{C}}$ as reference, the second-time colorization result $\mathbf{R}_{\mathbf{C}}^{\prime}$ should arrive back at $\mathbf{R}_{\mathbf{C}}$.

Based on this insight, we propose a new CNN model, named Cycle $\mathrm{CNN}$, that can be learned directly using the

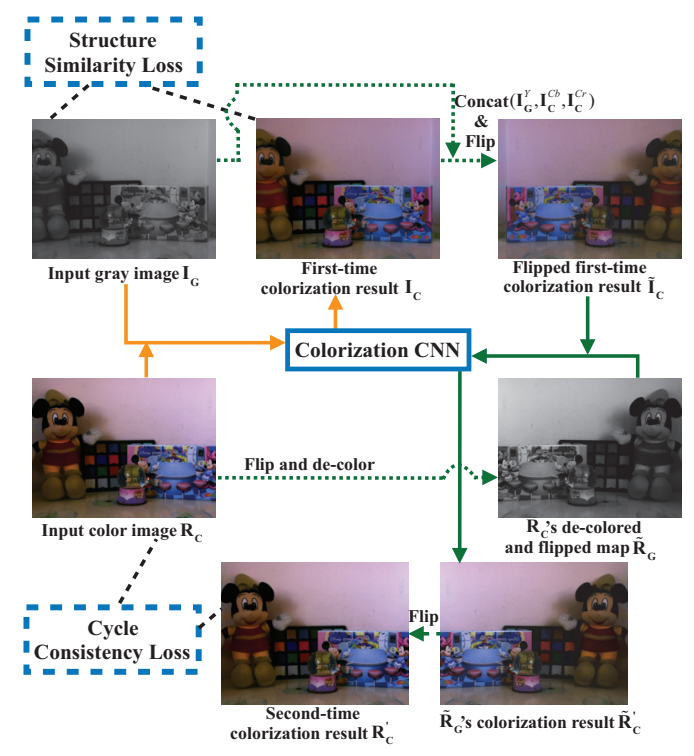

Figure 4: The overall structure of our Cycle CNN model.

real data from the monochrome-color system. In detail, as shown in Fig. 4, we use the colorization CNN to do the colorization twice, i.e. firstly colorizing $\mathbf{I}_{\mathbf{G}}$ using $\mathbf{R}_{\mathbf{C}}$ as reference and secondly colorizing $\mathbf{R}_{\mathbf{G}}$ using $\mathbf{I}_{\mathbf{C}}$ as reference. Our objective have three terms, i.e. structure similarity loss, cycle consistency loss, and spatial smoothness loss. For the first-time colorization result $\mathbf{I}_{\mathbf{C}}$, we introduce the structure similarity loss to encourage the structure similarity of the luminance maps between $\mathbf{I}_{\mathbf{G}}$ and $\mathbf{I}_{\mathbf{C}}$. For the second colorization result $\mathbf{R}_{\mathbf{C}}^{\prime}$, we introduce the cycle consistency loss to encourage the similarity of the color maps between $\mathbf{R}_{\mathbf{C}}$ and $\mathbf{R}_{\mathbf{C}}^{\prime}$. In addition, we introduce the spatial smoothness loss to encourage the spatial smoothness of the colorization result. We train the colorization $\mathrm{CNN}$ model by combining all these loses. We also use a refinement $\mathrm{CNN}$ to refine $\mathbf{I}_{\mathbf{C}}$ with $\mathbf{I}_{\mathbf{G}}$ as guidance to get the final result $\mathbf{I}_{\mathbf{C}}^{*}$.

Experimental results show that we can outperform related methods largely for the real data from the monochromecolor camera system.

Our contributions include 1) the cycle $\mathrm{CNN}$ structure that enables to train the model using real data from the monochrome-color camera systems, 2) the cycle consistency loss for the second-time colorization result, 3) the structure similarity loss for the first-time colorization result, and 4) the spatial smoothness loss for spatial smoothness of the colorization result.

\section{Related Works}

The existing colorization tasks can be divided into four kinds, i.e. automatic colorization, scribble-based colorization, reference-based colorization, and monochrome-color dual-lens colorization.

In automatic colorization, the input is only a single gray image and the algorithms need to automatically colorize it without any reference. Recent deep learning based methods, 
e.g. (Zhang, Isola, and Efros 2016) and (Iizuka, Simo-Serra, and Ishikawa 2016), make great progress to solve this problem. However, these methods are not proper for our problem because they fail to make use of the color image from the color camera, which contains much useful color information for colorizing the gray image from the monochrome camera.

In the scribble-based colorization task, the input includes a single gray image and several color scribbles which are drawn by humans. And the methods, e.g. (Zhang et al. 2017) and (Levin, Lischinski, and Weiss 2004), use the color scribbles as guidance to propagate the colors to the whole image. These methods are not suitable for our problem because there exist no scribbles in the monochrome-color camera system.

In the reference-based colorization task, the input includes an input gray image and a reference color image. Different from our problem, the reference image is shot in different locations and/or at different time and the contents within the pair of images just share similar semantics. Because the inputs are different from ours, the methods, e.g. (Welsh, Ashikhmin, and Mueller 2002; Ironi, Cohen-Or, and Lischinski 2005; Gupta et al. 2012; Furusawa et al. 2017; He et al. 2017; 2018; 2019), usually firstly search semantically similar pixels between the images and then propagate the colors of the matching pixels to the whole image. Welsh et al. (Welsh, Ashikhmin, and Mueller 2002) assume that pixels with the same grayscale intensity will have the same color, and use the luminance value as the feature to search for matching pixels. Ironi et al. (Ironi, Cohen-Or, and Lischinski 2005) use discrete cosine transform coefficients as the feature to search sparse matching pixels, copy the color of matching pixels for pixels in high confidence regions and then colorize pixels in low confidence regions by color propagation (Levin, Lischinski, and Weiss 2004). Gupta et al. (Gupta et al. 2012) extract features of superpixels by averaging feature values of all pixels among each superpixel, search for matching pixels by feature matching and use space voting for spatial consistency. Furusawa et al. (Furusawa et al. 2017) propose a referencebased colorization algorithm for colorizing manga images. The assumption for manga images are not always correct for general images. Thus, their results are not always good enough for solving our problem. He et al. (He et al. 2018; 2019) and Zhang et al. (Zhang et al. 2019) propose deep learning based algorithms for image and video colorization. But, they assume the pair of images are visually very different but semantically similar. Due to different assumptions from our problem, they do not consider locality and spatial smoothness and the proposed loss minimizes the semantic differences. Due to different loses, their results are not always faithful to the correct colors.

The monochrome-color dual-lens colorization task can be seen as a special case of reference-based colorization. Jeon et al. (Jeon et al. 2016) propose a stereo matching method to search for best-matching pixels, and correct colors in occlusion regions by applying spatial consistency of neighboring pixels over the whole image. Dong et al. (Dong et al. 2019) proposed a deep CNN for solving this problem and could achieve higher accuracy. However, they rely on synthesized

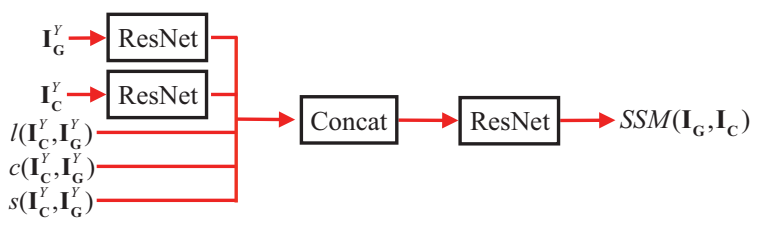

Figure 5: The structure of our structure similarity CNN model.

data to train the model. As discussed in the Introduction Section, the real data are quite different from the synthesized data, and their performances on the real data have big decrease.

Like sparseness, smoothness, etc., cycle consistency is also a marvelous and general property and can be utilized for solving different vision problems, e.g. image translation (Zhu et al. 2017), visual tracking (Wang, Jabri, and Efros 2019), super resolution (Wang et al. 2019), etc. In this paper, we make use of it for solving the colorization problem towards real monochrome-color camera systems.

Besides colorization, there exist some other enhancement problems in the multiple-camera system, like video retargeting (Li et al. 2018), deblur (Zhou et al. 2019), style transfer (Chen et al. 2018), etc. But, these methods cannot be directly used for our problem.

The difficulty of collecting ground-truth maps for training is also met in some other vision problems, e.g. depth estimation (Godard, Aodha, and Brostow 2017), image translation (Zhu et al. 2017), etc. And supervised learning methods are not always the most suitable solution. We share similar insights with these methods and propose a self-supervised colorization method in this paper.

\section{Overview}

\section{Method}

Our goal is to learn the colorization $\mathrm{CNN}$ model $M$ which maps any given pair of gray and reference color images $\mathbf{A}_{\mathbf{G}}$ and $\mathbf{B}_{\mathbf{C}}$ to the colorization result $\mathbf{A}_{\mathbf{C}}$, i.e. $M$ : $\left\{\mathbf{A}_{\mathbf{G}}, \mathbf{B}_{\mathbf{C}}\right\} \rightarrow \mathbf{A}_{\mathbf{C}}$. The training data are the real data from monochrome-color camera systems, as shown in Fig. 2.

As shown in Fig. 4, our cycle CNN framework does the colorization using the colorization $C N N M$ twice. First, we colorize the input gray image $\mathbf{I}_{\mathbf{G}}$ using the color image $\mathbf{R}_{\mathbf{C}}$ as reference. Second, we colorize the de-colored image of $\mathbf{R}_{\mathbf{C}}$, named $\mathbf{R}_{\mathbf{G}}$, using the first-time colorization result $\mathbf{I}_{\mathbf{C}}$ as reference. During the second-time colorization, we use the horizontally flipped maps of $\mathbf{R}_{\mathbf{G}}$ and $\mathbf{I}_{\mathbf{C}}$ to input the colorization CNN $M$ and do the flip for the result again to get the second-time colorization result $\mathbf{R}_{\mathbf{C}}^{\prime}$. It is because the model $M$ always search colors of pixels in the range of $(j, i)$ to $(j, i+d-1)$ in the reference image for each pixel $(j, i)$ in the input gray image, and the corresponding pixels in $\mathbf{I}_{\mathbf{C}}$ locate in the opposite search range, i.e. from $(j, i)$ to $(j, i-d+1)$. By doing the flip operations, we can enable the model $M$ to perform the second-time colorization without changing any model structure. Within the colorization $\mathrm{CNN}$ model, for any given pair of gray and reference color 


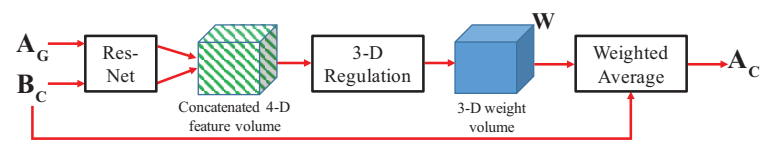

Figure 6: The colorization $\mathrm{CNN}$ model $M$ that colorize the given input gray image $\mathbf{A}_{\mathbf{G}}$ using the input color image $\mathbf{B}_{\mathbf{C}}$ as reference to obtain the colorization result $\mathbf{A}_{\mathbf{C}}$.

images $\mathbf{A}_{\mathbf{G}}$ and $\mathbf{B}_{\mathbf{C}}$, we follow (Dong et al. 2019) to learn the 3-D weight volume $\mathbf{W}$ between pixels of $\mathbf{A}_{\mathbf{G}}$ and $\mathbf{B}_{\mathbf{C}}$. And the colorization result $\mathbf{A}_{\mathbf{C}}$ is obtained by the weighted average operation between $\mathbf{W}$ and $\mathbf{B}_{\mathbf{C}}$. In the colorization CNN model, our objective have three terms, i.e. structure similarity loss, cycle consistency loss, and spatial smoothness loss. The structure similarity loss is to encourage the structure similarity of the luminance maps between input gray image $\mathbf{I}_{\mathbf{G}}$ and the first-time colorization result $\mathbf{I}_{\mathbf{C}}$. The cycle consistency loss is to encourage the similarity of the color maps between the reference color image $\mathbf{R}_{\mathbf{C}}$ and the second-time colorization result $\mathbf{R}_{\mathbf{C}}^{\prime}$. The spatial smoothness loss is to encourage the spatial smoothness of the colorization result.

We also use the refinement $\mathrm{CNN}$ to refine the first-time colorization result $\mathbf{I}_{\mathbf{C}}$ with $\mathbf{I}_{\mathbf{G}}$ as guidance to get the final result $\mathbf{I}_{\mathbf{C}}^{*}$.

\section{Structure similarity loss}

We propose the structure similarity loss to measure the structure similarity between the luminance maps of $\mathbf{I}_{\mathbf{C}}$ and $\mathbf{I}_{\mathbf{G}}$, i.e. $\mathbf{I}_{\mathbf{C}}^{Y}$ and $\mathbf{I}_{\mathbf{G}}^{Y}$. Our insight is that if the colorization is perfect, not only the color information, i.e. $\mathrm{Cb}$ and $\mathrm{Cr}$, should be correct, but also the luminance maps should have similar structures. $\mathbf{I}_{\mathbf{G}}^{Y}$ and $\mathbf{R}_{\mathbf{C}}^{Y}$ have different intensities, due to different luminance, blur, noise, etc., during the imaging process of the monochrome and color cameras. So, traditional loss metrics, e.g. L1 loss, L2 loss, etc, are not effective for our case.

We propose a CNN model to learn a deep metric to obtain the structure similarity loss between $\mathbf{I}_{\mathbf{G}}^{Y}$ and $\mathbf{I}_{\mathbf{C}}^{Y}$. As shown in Fig. 5, we use two ResNet to extract the features of $\mathbf{I}_{\mathbf{G}}^{Y}$ and $\mathbf{I}_{\mathrm{C}}^{Y}$ respectively. In addition, motivated by the success of SSIM (Wang et al. 2004), we also extract the luminance map $l$, the contrast map $c$ and the structure map $s$ between $\mathbf{I}_{\mathbf{G}}^{Y}$ and $\mathbf{I}_{\mathbf{C}}^{Y}$ as the features (please refer to more details in (Wang et al. 2004)). Then, we feed these five features to a ResNet to estimate the final structure similarity map $S S M$, and the structure similarity loss is obtained by

$$
L_{s s}=1-\mu\left(S S M\left(\mathbf{I}_{\mathbf{G}}, \mathbf{I}_{\mathbf{C}}\right)\right)
$$

where $\mu$ is the average operation.

To train the CNN model, we synthesize a dataset. First, we use the monochrome-color dual-camera system to shoot pairs of images in the distant view. Second, we use SIFT feature and projective transform to register the pairs of image. Among the registered results, we manually select and crop out the sub-regions where all pixels are perfectly registered and there exist no occlusions at all. The sub images

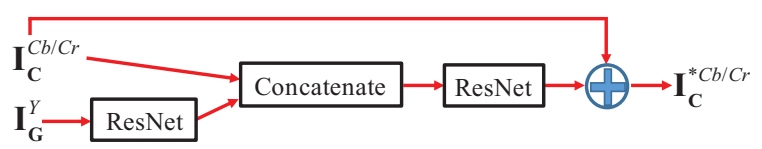

Figure 7: The refinement CNN model that refines the colorization result $\mathbf{I}_{\mathbf{C}}$ using the input gray image $\mathbf{I}_{\mathbf{G}}$ as guidance to obtain the final result $\mathbf{I}_{\mathrm{C}}^{*}$.

are named $\mathbf{J}_{\mathrm{G}}$ and $\mathbf{J}_{\mathbf{C}}$. Then, to simulate the structure distortions between $\mathbf{I}_{\mathbf{C}}^{Y}$ and $\mathbf{I}_{\mathbf{G}}^{Y}$, we propose to generate several random warping maps and use these maps to warp $\mathbf{J}_{\mathbf{G}}$ and $\mathbf{J}_{\mathbf{C}}$. The warped images are named $\dot{\mathbf{J}}_{\mathrm{G}}, \dot{\mathbf{J}}_{\mathbf{C}}$.

The pair of $\dot{\mathbf{J}}_{\mathbf{C}}$ and $\mathbf{J}_{\mathrm{G}}$ are used as the input, and the SSIM quality map (Wang et al. 2004) between $\dot{\mathbf{J}}_{\mathrm{G}}$ and $\mathbf{J}_{\mathrm{G}}$ is used as the ground-truth structure similarity map. We train the model using L1 loss. The random seeds include randomly values with sizes of $1 \times 1,2 \times 2,3 \times 3,4 \times 4,5 \times 5$, and we interpolate the seeds to the size of $\mathbf{J}_{\mathbf{G}}$ using Bicubic to obtain the random warping maps. The mean and variance of the random values of the seeds are 0 and $\{1,5,10,20,40\}$. In our colorization CNN model, we use the pre-trained structure similarity network to generate the structure similarity loss.

\section{Cycle consistency loss}

To encourage the similarity of the color maps between the reference color image $\mathbf{R}_{\mathbf{C}}$ and the second-time colorization result $\mathbf{R}_{\mathbf{C}}^{\prime}$, we propose the cycle consistency loss to measure the differences between $\mathbf{R}_{\mathbf{C}}$ and $\mathbf{R}_{\mathbf{C}}^{\prime}$. We use SSIM as the metric and we measure both $C b$ and $C r$ color channels, i.e.

$L_{c c}=1-\frac{1}{2}\left(S S I M\left(\mathbf{R}_{\mathbf{C}}^{C b}, \mathbf{R}_{\mathbf{C}}^{\prime C b}\right)+\operatorname{SSIM}\left(\mathbf{R}_{\mathbf{C}}^{C r}, \mathbf{R}_{\mathbf{C}}^{\prime C r}\right)\right)$,

\section{Spatial smoothness loss}

We introduce the spatial smoothness loss to encourage spatial smoothness of the $\mathbf{W}$ volume in the colorization CNN $M$ so as to obtain spatial smooth colorization result. We assume that neighboring pixels should have similar weights, so the loss is defined as

$$
L_{\text {smooth }}=\frac{1}{N} \sum_{(j, i, k)} \sum_{\left(j^{\prime}, i^{\prime}, k^{\prime}\right) \in \Omega(j, i, k)}\left|\mathbf{W}_{j, i, k}-\mathbf{W}_{j^{\prime}, i^{\prime}, k^{\prime}}\right| .
$$

where $\Omega$ is the 6-neighboring pixels in the three dimensions.

\section{Full objective}

Combining all above losses, the overall objective we aim to optimize is:

$$
L=\lambda_{1} L_{s s}+\lambda_{2} L_{c c}+\lambda_{3} L_{s m o o t h}
$$

where $\lambda_{1}, \lambda_{2}$, and $\lambda_{3}$ control the relative importance of the corresponding terms respectively. The values are set as $\lambda_{1}=$ $1, \lambda_{2}=1$, and $\lambda_{3}=0.1$ in this paper. With the guidance 

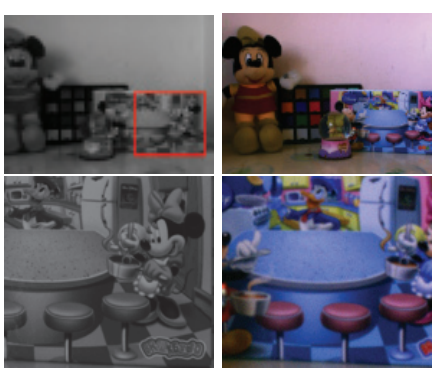

(a) Input gray and color images.

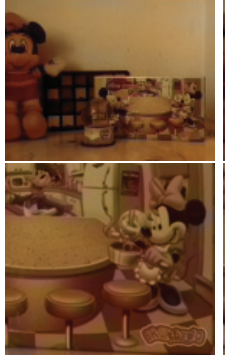

(b) Zhang et al.

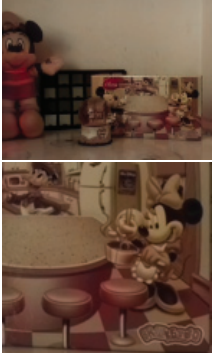

(c) Iizuka et al.

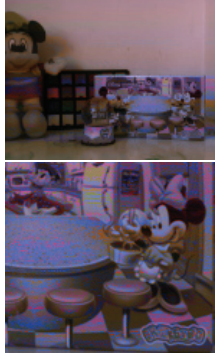

(d) Welsh et al.

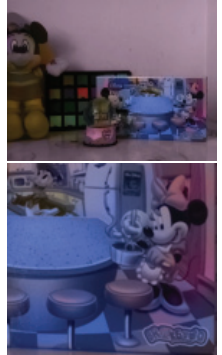

(e) Gupta et al.
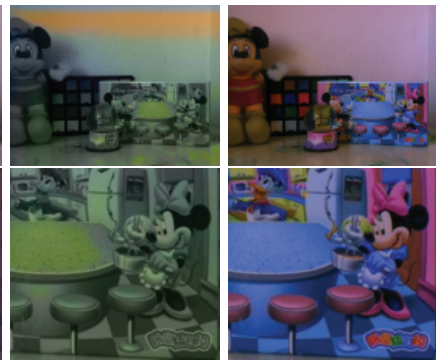

(f) Furusawa et al. (g) Ours.

Figure 8: Examples to compare the colorization results of the automatic colorization methods of Zhang et al., and lizuka et al., and the reference-based colorization methods of Welsh et al., Gupta et al., Furusawa et al., with ours.

of these losses, we successfully learn the colorization CNN without the ground-truth color information for training.

\section{Color refinement}

The first-time colorization result $\mathbf{I}_{\mathbf{C}}$ may have errors in occlusion regions. To correct these errors, we use the input gray image $\mathbf{I}_{\mathbf{G}}$ as guidance to refine the colorization result. We follow (Dong et al. 2019) to build the network. As shown in Fig. 7, the input gray image $\mathbf{I}_{\mathbf{G}}$ is fed into a ResNet to get its feature. The extracted feature and $\mathbf{I}_{\mathbf{C}}$ are concatenated and then fed into another ResNet to get the residue color map $\Phi\left(\mathbf{I}_{\mathbf{C}}, \mathbf{I}_{\mathbf{G}}\right)$. By adding $\mathbf{I}_{\mathbf{C}}$ and $\Phi\left(\mathbf{I}_{\mathbf{C}}, \mathbf{I}_{\mathbf{G}}\right)$, the final colorization result $\mathbf{I}_{\mathbf{C}}^{*}$ is obtained. To train this model, we use the second-time colorization results $\mathbf{R}_{\mathbf{C}}^{\prime}$ as inputs and the original input color image $\mathbf{R}_{\mathbf{C}}$ as ground-truth, and we use L2 loss for training.

\section{Network architecture}

We follow (Dong et al. 2019) to build the colorization CNN model and refinement $\mathrm{CNN}$ model. The pipeline is shown in Figs. 6 and 7. The ResNet has 18 convolution layers in total. The first residue block is with $5 \times 5$ kernel. The following 16 layers are 8 repeated residue blocks and each residue block consists of 2 convolution layers with $3 \times 3$ kernel and a residue connection. BatchNorm layers and $\mathrm{ReLu}$ layers are added after each convolution layer. The filter number $n$ of the 18 layers of ResNet is a hyper-parameter, which is set as 16 in this paper. 2) In the refinement module, the ResNets have similar network structure. The difference is that in the last layer the filter number is 1 and no BatchNorm layer or ReLu layer is added.

\section{Experimental Results}

\section{Dataset}

We use one monochrome camera and one color camera to shoot 1000 pairs of gray and color images to build the dataset, named Real Dataset. The monochrome and color cameras are rectified using the method of (Bradski and Kaehler 2008). The cameras are the monochrome and color versions of the same camera, i.e. the MVCAM-SU1000C camera.

\section{Implementation details}

The proposed deep convolutional network is implemented with TensorFlow. All models are optimized with RMSProp and a constant learning rate of 0.001 . We train with a batch size of 1 using a $256 \times 512$ randomly located crop from the input images. The images of the dataset is randomly divided into the training set, which contains 700 pairs of images, and the testing set, which contains 300 pairs of images. All the models are run on a server with an Intel I7 CPU and 4 NVIDIA Titan-X GPUs. The training time is about 15 hours and the testing time is about 0.4 seconds for $780 \times 1024$ test images.

\section{Comparison algorithms:}

We compare with state-of-the-art reference-based colorization algorithms, i.e. Welsh et al. (Welsh, Ashikhmin, and Mueller 2002), Ironi et al. (Ironi, Cohen-Or, and Lischinski 2005), Gupta et al. (Gupta et al. 2012), Furusawa et al. (Furusawa et al. 2017), He et al. 2018 (He et al. 2018), and He et al. 2019 (He et al. 2019), automatic colorization algorithms, i.e. Zhang et al. (Zhang, Isola, and Efros 2016) and Iizuka et al. (Iizuka, Simo-Serra, and Ishikawa 2016), and monochrome-color dual-lens colorization algorithms, i.e. Jeon et al. (Jeon et al. 2016) and Dong et al. (Dong et al. 2019).

\section{Comparison with other colorization methods on Real Dataset}

The qualitative results are shown in Figs. 8 and 9. As shown, our method has better results than the comparison methods. The automatic colorization methods (Iizuka, Simo-Serra, and Ishikawa 2016; Zhang, Isola, and Efros 2016) have wrong colors in most regions. It is because the input in these methods is only one single gray image, and the reference color image, which could provide much useful color information during the colorization, is not utilized at all. Welsh et al.'s method does not have good performance, because their assumption, i.e. pixels with the same grayscale intensity will have the same color value, is not true for many images. Gupta et al.'s method does not perform well, especially for objects with complicated textures. It is because the features of each superpixel are obtained by averaging the feature values of all pixels in the superpixel, which will 

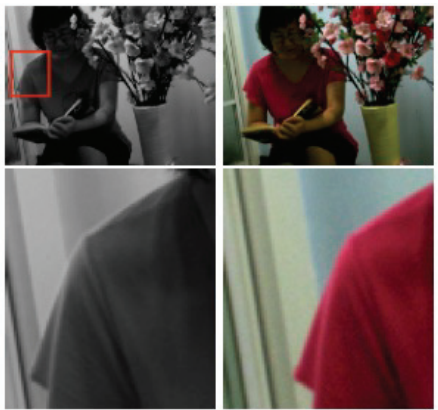

(a) Input gray and color images.

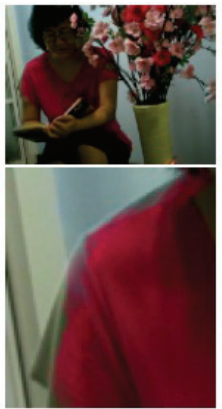

(b) Jeon et al.

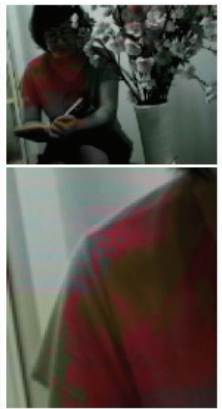

(c) Dong et al.

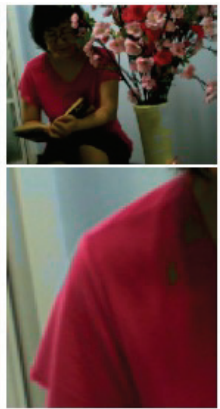

(d) Ironi et al.

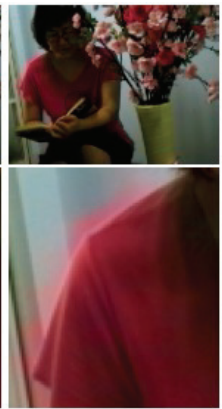

(e) He et al. 2018. (f) He et al. 2019

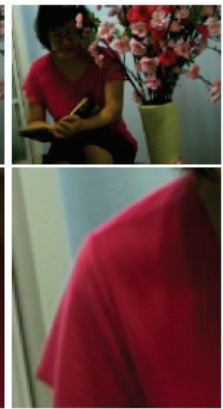

(g) Ours

Figure 9: Examples to compare the colorization results of the monochrome-color dual-lens colorization methods of Jeon et al., and Dong et al., and the reference-based colorization methods of Ironi et al., He et al. 2018, He et al. 2019, with ours.

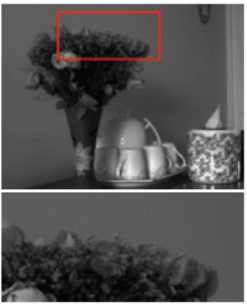

(a) Input gray and color images

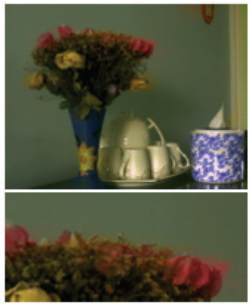

(b) No $L_{c c}$.

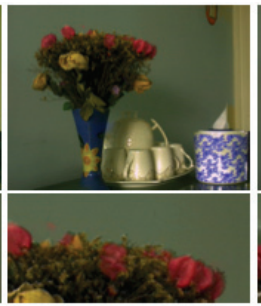

(c) No $L_{\text {smooth }}$.

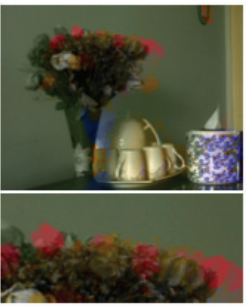

(d) No $L_{s s}$

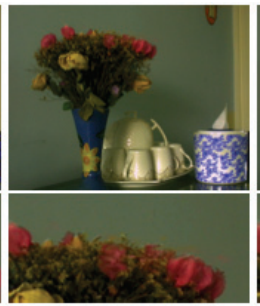

(e) SSIM for $L_{s s}$

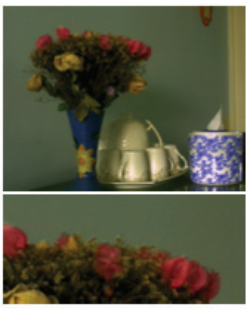

(f) Ours.

Figure 10: Example results in the ablation study.

decrease the accuracy of correspondence searching for our problem. Furusawa et al.'s result is not good enough because the method assumes that the images are manga images but in our problem the images are general images. Ironi et al.'s method has problems for edges and small objects because many unoccluded pixels are wrongly marked as occluded pixels, and thus the colorized pixels of unoccluded pixels are not enough for color propagation. Jeon et al.'s method is designed for monochrome-color system, but the handcrafted pipelines are not competing with our deep learning based model. Dong et al. have poor results because they are trained on synthesized data. Among the synthesized data, the pixels between the pair of gray and color images always have the same luminance and the distortions, e.g. blur and noise, are manually added. On the contrary, in the real data, the pixels between the pair of gray and color images usually have different luminance and the distortions are complicated and blind. Due to different characteristics of data in the synthesized and real datasets, Dong et al. have poor results for real data. He et al.'s results, including (He et al. 2018; 2019) could not achieve high accuracy. They are designed under the assumption that the pair of images are visually very different but semantically similar. Due to different assumptions from our problem, they do not consider locality and spatial smoothness and their losses minimize the semantic differences. Many pixels in the reference image may have similar semantics and thus this causes many inconsistent correspondence matches, which will cause wrong colorization.

A user study is also performed. There are 30 annotators

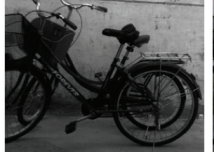

(a) Input pai

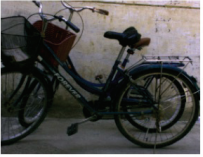
first-time and second-time results.

Figure 11: Examples of our first-time colorization results and second-time colorization results.

Table 1: Average SSIM values of the second-time colorization results of different methods on the Real Dataset. \begin{tabular}{|c|c|c|c|c|c|c|c|c|c|c|c|}
\hline & Welsh & Ironi & Gupta & Jeon & Furusawa & He18 & Zhang & lizuka & Dong & He19 & Ours \\
\hline SSIM & 0.861 & 0.892 & 0.872 & 0.906 & 0.841 & 0.898 & 0.831 & 0.851 & 0.871 & 0.902 & $\mathbf{0 . 9 5 4}$ \\
\hline
\end{tabular}

in total. The annotation choices include five score level, i.e. 'Perfect', 'Few Errors', 'Partly Wrong', 'Mostly Wrong', and 'Totally Wrong'. The annotators are asked to annotate every colorization result of the 10 comparing methods and ours. The whole set of images in the user study are 100 pairs that are randomly selected from our Real Dataset. And each annotator annotates 1100 colorization results in total. To avoid outlier annotation, we will let each annotator randomly re-annotate some results and see the annotations as outlier if the annotation differences are beyond one score level. The results are shown in Fig. 12. This shows we can get 'Perfect' and 'Few Errors' scores in most cases and our method gets much higher perceptual scores than the others.

Objective evaluation is also performed by evaluating 


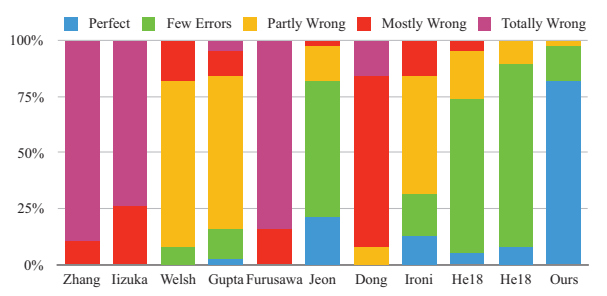

Figure 12: User study results.

Table 2: Average SSIM values of the first-time colorization results and the second-time colorization results of different methods on the four synthesized datasets. CT, MB, ST, and SF are short for the datasets of Cityscapes, Middlebury, Sintel, and SceneFlow, respectively

\begin{tabular}{|c|c|c|c|c|c|c|c|c|}
\hline & \multicolumn{3}{|c|}{ First-time colorization } & \multicolumn{3}{c|}{ Second-time colorization } \\
\hline & CT & MB & ST & SF & CT & MB & ST & SF \\
\hline Welsh & 0.897 & 0.906 & 0.795 & 0.813 & 0.907 & 0.948 & 0.819 & 0.836 \\
\hline Ironi & 0.897 & 0.940 & 0.918 & 0.890 & 0.901 & 0.947 & 0.923 & 0.897 \\
\hline Gupta & 0.948 & 0.896 & 0.933 & 0.869 & 0.951 & 0.902 & 0.945 & 0.883 \\
\hline Jeon & 0.953 & 0.958 & 0.943 & 0.927 & 0.953 & 0.950 & 0.959 & 0.928 \\
\hline Furusawa & 0.841 & 0.860 & 0.794 & 0.795 & 0.842 & 0.869 & 0.789 & 0.797 \\
\hline He18 & 0.951 & 0.949 & 0.948 & 0.919 & 0.963 & 0.952 & 0.956 & 0.924 \\
\hline Zhang & 0.460 & 0.746 & 0.687 & 0.279 & 0.435 & 0.723 & 0.691 & 0.294 \\
\hline Iizuka & 0.757 & 0.677 & 0.852 & 0.411 & 0.758 & 0.681 & 0.851 & 0.413 \\
\hline Dong & 0.982 & 0.981 & 0.983 & 0.988 & 0.986 & 0.984 & 0.986 & 0.991 \\
\hline He19 & 0.952 & 0.953 & 0.947 & 0.921 & 0.958 & 0.951 & 0.953 & 0.923 \\
\hline Ours & $\mathbf{0 . 9 8 3}$ & $\mathbf{0 . 9 8 3}$ & $\mathbf{0 . 9 8 4}$ & $\mathbf{0 . 9 8 9}$ & $\mathbf{0 . 9 8 7}$ & $\mathbf{0 . 9 8 4}$ & $\mathbf{0 . 9 8 8}$ & $\mathbf{0 . 9 9 2}$ \\
\hline
\end{tabular}

the SSIM values between the second-time colorization results and the input color image. Due to the lack of groundtruth color information of input gray images, we cannot perform objective evaluation for the first-time colorization results. But, according to the cycle consistency property, the second-time colorization results can still reflect the colorization quality to some extend. So we use all the methods to do the colorization twice. The results are in Table 1, which show that we outperform largely than the other methods.

\section{Comparison with other colorization methods on Synthesized Dataset}

Due to the importance of objective evaluation, we perform our method on the traditional synthesized dataset of (Dong et al. 2019; Jeon et al. 2016). The datasets include Cityscapes (Cordts et al. 2016), Middlebury (Scharstein and Pal 2007), Sintel (Butler et al. 2012), and SceneFlow (Mayer et al. 2016). We use all the comparing methods and ours to do the colorization twice, and the objective results are shown in Table 2. As shown, on the four synthesized datasets, we could still have higher results than all the other methods. The reason is that, although most of our colorization CNN and refinement CNN models are similar with (Dong et al. 2019), we add the spatial smoothness loss into the colorization CNN model and the cycle colorization structure, i.e. colorizing twice, actually augments the training data in two times. We also test the linear correlation coefficients (LCC) between our first-time colorization results and second-time colorization results on the four datasets. The results are shown in Table 3. As shown, they have very high correlation. This verifies our insight of cycle colorization consis-
Table 3: LCC between the SSIM values of our first-time colorization results and our second-time colorization results on the four synthesized datasets.

\begin{tabular}{|c|c|c|c|c|}
\hline & CT & MB & ST & SF \\
\hline LCC & 0.9884 & 0.9844 & 0.9915 & 0.9917 \\
\hline
\end{tabular}

Table 4: Ablation study. We show average SSIM values of the second-time colorization results of different variants of our model on the Real Dataset.

\begin{tabular}{|c|c|}
\hline & SSIM \\
\hline No cycle consistency loss & 0.8140 \\
\hline No structure similarity loss & 0.9192 \\
\hline No spatial smoothness loss & 0.9432 \\
\hline SSIM as structure similarity loss & 0.9236 \\
\hline Ours & $\mathbf{0 . 9 5 4 7}$ \\
\hline
\end{tabular}

tency and provide support that the results in Table 1 could reflect the colorization quality of different methods.

\begin{abstract}
Ablation study
The ablation study compares a number of different model variants and justifies our design choices. We wish to evaluate the importance of the key ideas in this paper: the cycle consistency loss, the structure similarity loss, the spatial smoothness loss. So we remove each of these losses and retrain the model. Table 4 shows the summary performance of different model variants. Fig. 10 shows some subjective examples. The results show that any of these variants will degrade the colorization accuracy. This verifies the contributions of all these losses.
\end{abstract}

\section{Conclusions}

We have presented a novel CNN model for colorization in real monochrome-color dual-lens system. It can be trained directly using the real data from monochrome-color camera systems. The proposed method uses the CNN model to do the colorization twice. In addition, we introduce the cycle consistency loss, the structure similarity loss, and the spatial smoothness loss. Our method achieves superior performance than the state-of-the-art methods for colorizing real data.

\section{Acknowledgments}

This work is funded by the National Nature Science Foundation of China (No. 61802026 and 61806016) and the Fundamental Research Funds for the Central University (No. 2019RC39). We thank the anonymous reviewers for helping us to improve this paper.

\section{References}

Bradski, G., and Kaehler, A. 2008. Learning opencv : Computer vision with the opencv library.

Butler, D. J.; Wulff, J.; Stanley, G. B.; and Black, M. J. 2012. A naturalistic open source movie for optical flow evaluation. European Conference on Computer Vision 611-625. 
Chen, D.; Yuan, L.; Liao, J.; Yu, N.; and Hua, G. 2018. Stereoscopic neural style transfer. The IEEE Conference on Computer Vision and Pattern Recognition.

Cordts, M.; Omran, M.; Ramos, S.; Rehfeld, T.; Enzweiler, M.; Benenson, R.; Franke, U.; Roth, S.; and Schiele, B. 2016. The cityscapes dataset for semantic urban scene understanding. IEEE Conference on Computer Vision and Pattern Recognition 3213-3223.

Dong, X.; Li, W.; Wang, X.; and Wang, Y. 2019. Learning a deep convolutional network for colorization in monochrome-color dual-lens system. AAAI Conference on Artificial Intelligence.

Furusawa, C.; Hiroshiba, K.; Ogaki, K.; and Odagiri, Y. 2017. Comicolorization: semi-automatic manga colorization. SIGGRAPH Asia.

Godard, C.; Aodha, O.; and Brostow, G. 2017. Unsupervised monocular depth estimation with left-right consistency. $C V P R$.

Gupta, R. K.; Chia, A. Y. S.; Rajan, D.; Ng, E. S.; and Zhiyong, H. 2012. Image colorization using similar images. ACM international conference on Multimedia 369-378.

He, M.; Liao, J.; Yuan, L.; and Sander, P. 2017. Neural color transfer between images. Arxiv.

He, M.; Chen, D.; Liao, J.; Sander, P.; and Yuan, L. 2018. Deep exemplar-based colorization. ACM SIGGRAPH.

He, M.; Liao, J.; Chen, D.; Yuan, L.; and Sander, P. 2019. Progressive color transfer with dense semantic correspondences. ACM Transactions on Graphics 38(13).

Iizuka, S.; Simo-Serra, E.; and Ishikawa, H. 2016. Let there be color!: joint end-to-end learning of global and local image priors for automatic image colorization with simultaneous classification. ACM Transactions on Graphics 35(4).

Ironi, R.; Cohen-Or, D.; and Lischinski, D. 2005. Colorization by example. Rendering Techniques 201-210.

Jeon, H. G.; Lee, J. Y.; Im, S.; Ha, H.; and Kweon, I. S. 2016. Stereo matching with color and monochrome cameras in low-light conditions. IEEE Conference on Computer Vision and Pattern Recognition 4086-4094.

Levin, A.; Lischinski, D.; and Weiss, Y. 2004. Colorization using optimization. ACM transactions on graphics 23(3):689-694.

Li, B.; Lin, C.; Shi, B.; Huang, T.; Gao, W.; and Kuo, C. 2018. Depth-aware stereo video retargeting. CVPR.

Mayer, N.; Ilg, E.; Hausser, P.; Fischer, P.; D.Cremers; A.Dosovitskiy; and Brox, T. 2016. A large dataset to train convolutional networks for disparity, optical flow, and scene flow estimation. IEEE Conference on Computer Vision and Pattern Recognition 4040-4048.

Scharstein, D., and Pal, C. 2007. Learning conditional random fields for stereo. IEEE Conference on Computer Vision and Pattern Recognition 1-8.

Wang, Z.; Bovik, A. C.; Sheikh, H.; and Simoncelli, E. P. 2004. Image quality assessment from error visibility to structural similarity. IEEE Transactions on Image Processing 13(4):600-612.
Wang, L.; Wang, Y.; Liang, Z.; Lin, Z.; Yang, J.; An, W.; and Guo, Y. 2019. Learning parallax attention for stereo image super-resolution. CVPR.

Wang, X.; Jabri, A.; and Efros, A. 2019. Learning correspondence from the cycle-consistency of time. CVPR.

Welsh, T.; Ashikhmin, M.; and Mueller, K. 2002. Transferring color to greyscale images. ACM transactions on graphics 21(3):277-280.

Zhang, R.; Zhu, J.; Isola, P.; Geng, X.; Lin, A.; Yu, T.; and Efros, A. 2017. Real-time user-guided image colorization with learned deep priors. ACM Transactions on Graphics 36(4).

Zhang, B.; He, M.; Liao, J.; Sander, P.; Yuan, L.; Bermak, A.; and Chen, D. 2019. Deep exemplar-based video colorization. CVPR.

Zhang, R.; Isola, P.; and Efros, A. 2016. Colorful image colorization. European Conference on Computer Vision 649666.

Zhou, S.; Zhang, J.; Zuo, W.; Xie, H.; Pan, J.; and Ren, J. 2019. Davanet: Stereo deblurring with view aggregation. CVPR.

Zhu, J.; Park, T.; Isola, P.; and Efros, A. 2017. Unpaired image-to-image translation using cycle-consistent adversarial networks. ICCV. 